\title{
Labelling Heuristics for CSP Application Domains
}

\author{
Zeynep Kizıltan \\ Computer Science Division, Department of Information Science \\ Uppsala University, Box 513, S - 75120 Uppsala, Sweden \\ Zeynep.Kiziltan@dis.uu.se
}

Many real-life problems are constraint satisfaction problems (CSPs), which can be programmed as constraint models and then be solved using constraint solvers. Constraint solvers are equipped with a search algorithm, such as forwardchecking, and labelling heuristics, one of which is the default. To enhance the performance of constraint models, a lot of research has been made in recent years to develop new labelling heuristics, which concern the choice of the next variable to branch on during the search and the choice of the value to be assigned to that variable. These heuristics significantly reduce the search space.

However, little is said about the application domains of these heuristics, so modellers find it difficult to decide when to apply a particular heuristic, and when not. Indeed, it is not a trivial task to infer the application domains of heuristics because the performance of heuristics is not only model-dependent but also instance-dependent, i.e., for a given constraint model, a heuristic can perform well for some (distributions on the) instances, but very poorly on others; this is taken into account by some generators of model-specific solvers.

Instead of inferring the application domains of heuristics, we advocate inferring heuristics for application domains. If a mapping between application domains and heuristics is known to the solver, then modellers can - if they wish so - be relieved from the procedural aspect of modelling, namely figuring out which heuristic to indicate or implement. Forcing modellers to deal with this procedural aspect may not only add a challenging step but also has the disadvantage that they must commit - at modelling time - to a single heuristic and thus expose their models to the instance sensitivity of heuristics.

Towards inferring labelling heuristics for application domains, our three-step approach is to first formalise an application domain as a family of CSP models, so as to exhibit the generic constraint store for all models in that family. Second, the interaction - for a given search algorithm - between the constraints in this generic store and the domain propagation during search is analysed, so as to infer - before modelling any CSP — suitable heuristics for any model in that family. Due to the instance sensitivity of heuristics, the outcome of this process usually is a set of heuristics, rather than a single one. The final step of our approach is to address the issue of selecting or switching — at solving time — among the inferred family-specific heuristics, according to the instance to be solved.

Our ultimate aim is thus a new generation of more intelligent solvers that allow CSP modellers to concentrate on the declarative aspect of modelling, without compromising (much) on efficiency. 